is actually impossible to beat (even in the most improbable world), whereas for deterministic processes any rate faster than linear inevitably leads to a vanishing fidelity. Interestingly, these bounds are provided by establishing close links between probabilistic cloning and quantum metrology ${ }^{8}$.

However, spreading quantum information at such high rates comes at a price: it happens only rarely with an exponentially small probability as $N$ increases. What importance shall we give to such exponentially deprived events? It depends on what is at stake. Favourable genetic mutations, earthquakes and super-replication are all improbable. However, each of these events has drastic consequences and one should avoid undermining their existence. Besides assessing their odds, it is critical to be able to identify those events when they occur so as to take the necessary actions. Heralded events, such as super-replication, can then easily function as primitives in more complex procedures. At a more fundamental level, it is important to distinguish between ultimate versus de facto quantum limits. No matter how unlikely an event is, once it occurs it is a certainty; and certainties cannot violate ultimate bounds. Moreover, if we stick to the operational definition of reality as something that can kick back, then a rare but 'hard-kicking' event deserves a place in our description of reality.

It remains an open question whether super-replication is feasible with current technology. In this respect it is very plausible that the super-replication regime can only be attained for moderate values of $N$, as the Heisenberg limit is often downgraded to the standard quantum limit in the presence of noise as $N \rightarrow \infty$. As a closing remark, although the quantum clock-cloner can superreplicate very occasionally, I would safely bet that in the unsuccessful events the original copies will mostly remain untouched. Hence, super-replication is a spectacular and rare endeavour that entails little risk.

John Calsamiglia is at Física Teòrica:

Informació i Fenòmens Quàntics, Departament de Física, Universitat Autònoma de Barcelona, Bellaterra 08193, Spain.

e-mail:John.Calsamiglia@uab.cat

\section{References}

1. Chiribella, G. et al. Nature Commun. 4, 2915 (2013).

2. Scarani, V. et al. Rev. Mod. Phys. 77, 1225-1256 (2005).

3. Aaronson, S. in The Once and Future Turing: Computing the World (eds Cooper, S. B. \& Hodges, A.) Ch. 12

(Cambridge Univ. Press, 2014).

4. Bužek, V. \& Hillery, M. Phys. Rev. A 54, 1844-1852 (1996).

5. Fiurášek, J. Phys. Rev. A 70, 032308 (2004).

6. Duan, L.-M. \& Guo, G.-C. Phys. Rev. Lett. 80, 4999-5002 (1998),

7. Ralph, T. C. \& Lund, A. in Proc. 9th Int. Conf. Quantum Communication Measurement and Computing (ed. Lvovsky, A.) 155-160 (AIP, 2009)

8. Gendra, B. et al. Phys. Rev. Lett. 110, 100501 (2013).

Published online: 5 January 2014

\title{
GALACTIC CENTRE
}

\section{The final countdown}

All eyes are on a cloud of gas, known as G2, that is spiralling towards its doom at the centre of the Milky Way.

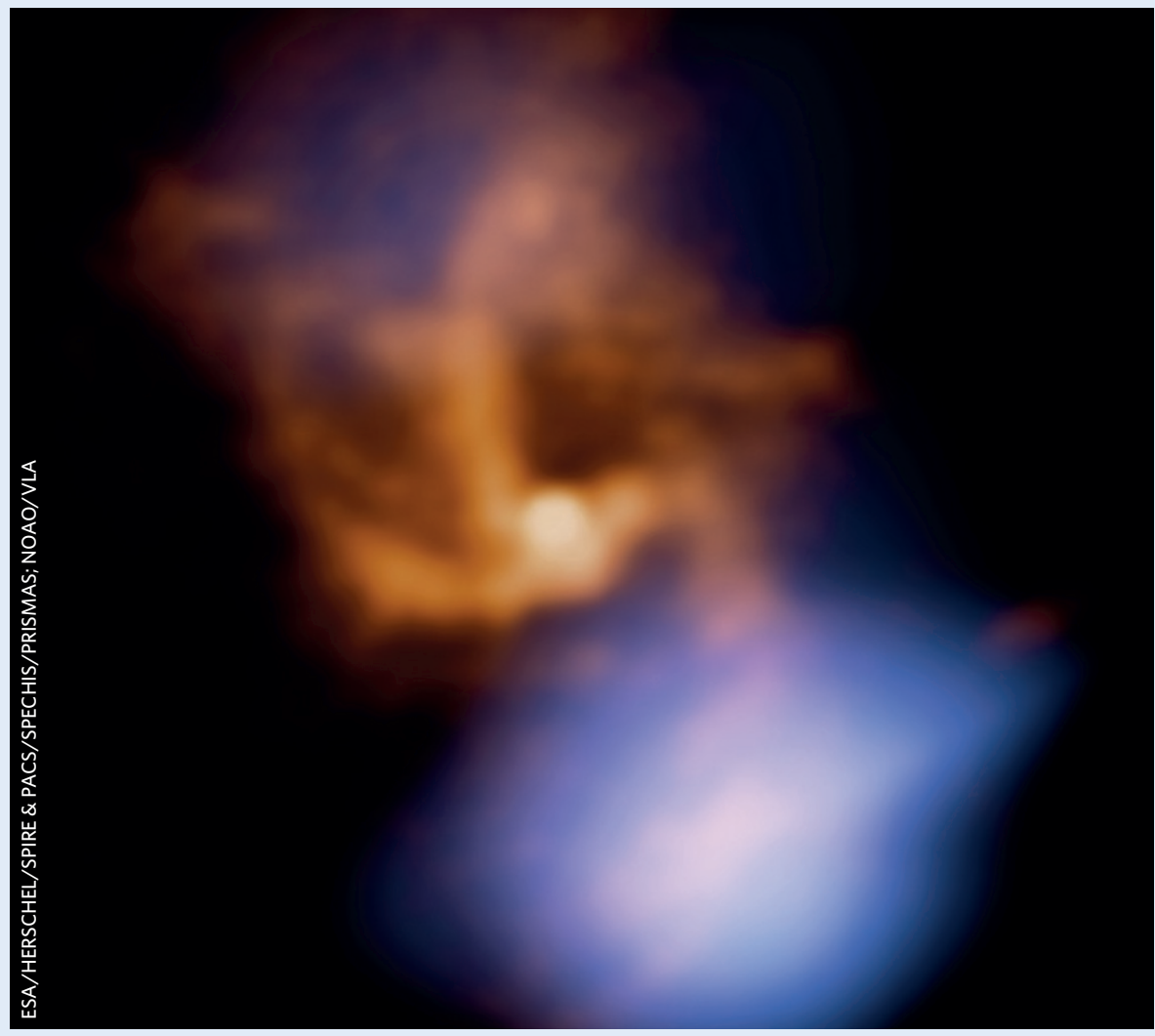

Discovered in 2011 and with a mass that is three times that of the Earth, G2 is approaching the supermassive black hole, Sagittarius $A^{\star}$ - the bright spot in this image from ESA's Herschel space observatory; the blue region shows gas within 10 light-years of the black hole, with hot infalling gas appearing orange. Astronomers first thought the encounter would occur in late 2013, but now estimate the show will begin in early 2014.

Owing to dust obscuring $\mathrm{G} 2$ as it moves rapidly away along our line of sight, the exact nature of the cloud remains unclear. If it is indeed a gas cloud, as originally reported, it was probably formed by the collision of stellar winds. However, some evidence suggests it may be an unresolved star. In any case, the onset of tidal shearing, due to gravitational interactions with the tidal field of the black hole, is already measurable.

Although many theories and predictions exist for the fate of $\mathrm{G} 2$ (summarized in a preprint by Stefan Gillessen et al., http://arxiv.org/abs/1312.4386; 2013), only time will tell. A gas cloud would be completely disrupted by Sagittarius $A^{*}$, possibly creating a glow for a year or more and/or a large flare; a star, however, could escape unharmed and subsequently reform its gas cover. NASA's Swift space telescope is providing daily updates in the X-ray band, where the most stunning changes will occur.

MAY CHIAO 